\title{
Relações entre Burnout, Traços de Personalidade e Variáveis Sociodemográficas em Trabalhadores Brasileiros
}

\author{
Vitor Hugo Loureiro Bruno Costa ${ }^{1}$ \\ Juliane Callegaro Borsa ${ }^{2}$ \\ Bruno Figueiredo Damásio ${ }^{3}$ \\ ${ }^{1}$ Universidade Estácio de Sá, Rio de Janeiro, Rio de Janeiro, Brasil \\ ${ }^{2}$ Pontifícia Universidade Católica do Rio de Janeiro,Rio de Janeiro, Rio de Janeiro, Brasil \\ ${ }^{3}$ Universidade Federal do Rio de Janeiro, Rio de Janeiro, Rio de Janeiro, Brasil
}

\begin{abstract}
Resumo
O Burnout é caracterizado pela exaustão emocional, despersonalização e baixa realização no trabalho. O objetivo desta pesquisa foi investigar as relações entre variáveis sociodemográficas e traços de personalidade, segundo o modelo dos Cinco Grandes Fatores, no desfecho da síndrome. Os seguintes questionários: sociodemográfico, Inventário de Burnout no Trabalho e Marcadores Reduzidos de Personalidade foram respondidos, em plataforma on-line, por 343 profissionais brasileiros do setor de serviços (75,50\% mulheres, $n=259)$ atuantes nas áreas da saúde, educação, serviços administrativos, segurança, bancários e atendimento ao público (e.g., telemarketing, call centers). Os resultados não apontam para relações estatisticamente significativas entre as variáveis sociodemográficas e o Burnout. Os traços de personalidade desempenharam relação preditiva mais relevante com os três fatores do Burnout, sendo o Neuroticismo o maior preditor. A partir dos dados, pode-se dizer que os recursos pessoais podem exercer um importante papel no desenvolvimento do Burnout, com especial atenção ao Neuroticismo.

Palavras-chave: stress ocupacional, traços de personalidade, demografia, qualidade de vida no trabalho
\end{abstract}

Relationship between Burnout, Personality Traits and Sociodemographic Variables in Brazilian Workers

\begin{abstract}
Burnout is characterized by emotional exhaustion, depersonalization, and low personal accomplishment at work. The objective of this research was to investigate the relationship between sociodemographic variables and personality traits, according to the Big Five theory, in the outcome of the syndrome. The sociodemographic questionnaire, the Work Burnout Inventory and the Reduced Personality Markers were answered in an online platform by 343 Brazilian professionals from the service sector ( $75.50 \%$ women, $n=259)$, working in the areas of health, education, administrative services, security, banking and customer service (e.g. telemarketing, call centers). The results do not point to a statistically significant relationship between sociodemographic variables and burnout. Personality traits had a more predictive relationship with the three factors of burnout, which Neuroticism was the most important predictor. Personal resources, especially Neuroticism, can play an important role in the development of burnout.

Keywords: occupational stress; personality traits; demographic characteristics; quality of work life
\end{abstract}

\section{Relaciones entre Burnout, Rasgos de Personalidad y Variables Sociodemográficas en Trabajadores Brasileños}

\section{Resumen}

El burnout se caracteriza por el agotamiento emocional, la despersonalización y la reducción de la realización en el trabajo. El objetivo de esta investigación fue investigar las relaciones entre variables sociodemográficas y rasgos de personalidad, según el modelo de los Cinco Grandes Factores, en el desenlace del síndrome. El cuestionario sociodemográfico, el Inventario de Burnout en el Trabajo y los Marcadores Reducidos de la Personalidad fueron contestados, en una plataforma digital, por 343 profesionales brasileños $(75,50 \%$ mujeres, $n=259)$ en el sector de servicios (p. ej., áreas de salud, educación, seguridad). Los resultados no apuntan a una relación estadísticamente significativa entre las variables sociodemográficas y el burnout. Los rasgos de personalidad desempeñaron relación predictiva más relevante con los tres factores del burnout, siendo el Neuroticismo el principal predictor. Fue observado que los recursos personales pueden desempeñar un importante papel en el desarrollo del burnout, con especial atención al Neuroticismo.

Palabras clave: estrés ocupacional; rasgos de personalidad; demografía; calidad de vida en el trabajo

O burnout tem sido compreendido como uma resposta aos estressores crônicos interpessoais e emocionais experienciados pelo indivíduo no seu ambiente de trabalho (Maslach \& Leiter, 2016; Maslach, Schaufeli, \& Leiter, 2001). Devido aos efeitos negativos desta síndrome, tanto nos indivíduos quanto nas organizações (Shanafelt et al, 2012), diversos pesquisadores buscam compreender quais são os aspectos pessoais e contextuais que auxiliam no desenvolvimento, manutenção e agravamento dos seus sintomas (Laschinger \& 
Fida, 2014; Purvanova \& Muros, 2010; Swider \& Zimmerman, 2010; You, Huang, Wang, \& Bao, 2015).

Maslach e Jackson (1981) foram as primeiras autoras a operacionalizarem o burnout como um construto composto por três dimensões distintas, mas relacionadas entre si: a Exaustão Emocional, a Despersonalização e a Baixa Realização no Trabalho. A Exaustão Emocional é compreendida como uma sensação de esgotamento mental e físico, na qual o indivíduo acredita não mais dispor da energia e vitalidade que antes possuía para desempenhar suas tarefas no cotidiano do trabalho (Maslach \& Leiter, 2016). A Despersonalização, também reportada na literatura como cinismo, é a dimensão interpessoal da síndrome e refere-se a respostas insensíveis e impessoais, aparentando certo distanciamento para com os colegas de trabalho, clientes e, inclusive, para com a instituição em que trabalha (Maslach et al., 2001). E, por fim, a Baixa Realização no Trabalho se refere à tendência de o profissional avaliar-se negativamente, julgando-se incompetente perante sua atuação profissional (Maslach \& Jackson, 1981).

Os fatores relacionados ao burnout são muitos, sendo, em geral, agrupados em fatores ocupacionais, organizacionais e individuais (Maslach et al., 2001; Shirom, 2003). Os estudos precursores sobre o burnout focavam, majoritariamente, nos grupos profissionais das áreas assistencialistas (e.g. saúde, educação, assistência social), uma vez que acreditava-se que estas ocupações tinham características facilitadoras para o esgotamento profissional, a saber: (1) contato direto e constante com problemas físicos, emocionais e psicológicos do público; (2) exposição prolongada a altos níveis de estresse; e (3) a crença de que, normalmente, as pessoas que escolhiam tais profissões estavam mais propensas a se desiludirem com a realidade das dificuldades do trabalho (Freudenberger, 1974). No entanto, estudos posteriores apontam que mais importante que o viés ocupacional, isto é, as características peculiares a cada categoria profissional, a forma disfuncional como o trabalho está estruturado (e.g. sobrecarga de trabalho, baixa autonomia, poucas recompensas, conflitos interpessoais) caracterizam os fatores organizacionais capazes de melhor explicar o surgimento e agravamento dos sintomas (Demerouti, Nachreiner, Bakker, \& Schaufeli, 2001; Maslach \& Leiter, 1997; Maslach, Schaufeli, \& Leiter, 2001; Maslach \& Leiter, 2016).

Por conta dos impactos organizacionais e o interesse em compreendê-los, o foco das pesquisas sobre o burnout tem sido acerca dos fatores contingenciais que permeiam as organizações, das peculiaridades do fazer profissional de cada ocupação, deixando-se à margem fatores individuais, como os traços de personalidade e variáveis sociodemográficas (Bostjancic, Kocjan, \& Stare, 2015; Swider \& Zimmerman, 2010). Pesquisar o papel desses fatores no desfecho do burnout é importante para se ter uma maior compreensão do perfil de vulnerabilidade dos indivíduos afetados pela síndrome (Campos, Angélico, Oliveira, \& Oliveira, 2015).

\section{Traços de personalidade e o Burnout}

O modelo teórico de Recursos e Demandas no Trabalho (Demerouti, Nachreiner, Bakker, \& Schaufeli, 2001), o qual entende que as tensões emocionais num dado ambiente laboral são fruto do desequilíbrio entre as altas demandas da organização (e.g. carga de trabalho, pressão por produtividade) sobre o funcionário e os recursos por ela disponibilizados para lidar com essas demandas (e.g. feedback, progressão de carreira), tem sido bastante aceito para se compreender os fatores que levam ao desfecho do burnout no trabalho (Bakker, Demerouti, \& Sanz-Vergel, 2014; Demerouti et al., 2001). Recentemente, Schaufeli e Taris (2014) acrescentaram ao modelo original a variável de recursos pessoais, referindo-se às características psicológicas próprias do indivíduo capazes de impactar seu ambiente de trabalho, como os traços de personalidade, por exemplo (Dalanhol, Freitas, Machado, Hutz, \& Vazquez, 2017).

A partir do enfoque teórico do modelo dos Cinco Grandes Fatores (CGF), a personalidade pode ser entendida por meio de cinco fatores independentes, mas inter-relacionados, a saber: Extroversão, Abertura à experiência, Socialização, Conscienciosidade e Neuroticismo. Os traços de personalidade podem interferir tanto nos padrões comportamentais e emocionais do indivíduo quanto no desempenho, bem-estar e saúde mental apresentado por ele(a) em seu trabalho (Dalanhol et al., 2017; Judge, Higgins, Thoresen, \& Barrick, 1999).

O fator de Extroversão descreve até que ponto as pessoas são assertivas, dominantes e comunicativas, tendendo a experienciar mais emoções positivas, como otimismo e entusiasmo, e a buscarem excitação na interação com outras pessoas (Judge et al., 1999). Por apresentarem emoções positivas, espera-se que pessoas com altos níveis de extroversão sejam mais esperançosas quanto ao futuro e tenham mais recursos para manterem um bom desempenho em suas ocupações e um bom relacionamento com seus pares (Judge \& Ilies, 
2002) e, comumente, é encontrada relação negativa entre esse traço de personalidade e os três fatores do burnout (Swider \& Zimmerman, 2010).

O Neuroticismo, por sua vez, é um traço caracterizado por uma maior tendência à instabilidade emocional, ansiedade e humor deprimido (Ashton, 2013). A instabilidade emocional e a propensão ao pessimismo dos indivíduos com elevados níveis desse traço dificultam o emprego de estratégias adequadas para lidar com um ambiente de trabalho de muitas demandas, sendo estes mais suscetíveis ao adoecimento psíquico no trabalho (Huang, Ryan, Zabel, \& Palmer, 2014). Estudos apontam para o Neuroticismo como sendo o traço de personalidade mais relacionado ao burnout e relacionando-se significativamente a todos os fatores da síndrome (Swider \& Zimmerman, 2010).

A Abertura à experiência está atrelada às pessoas mais flexíveis, intelectualmente curiosas, que dão preferência às soluções mais criativas do que às mais superficiais e tradicionais (Ashton, 2013). Pessoas com maior tendência à Abertura à experiência normalmente apresentam bom desempenho no trabalho, no entanto, a dificuldade em se sujeitar a determinadas condições da própria organização pode resultar em problemas a nível de relacionamento interpessoal (Heineck \& Anger, 2010).

Já o traço de Socialização diz respeito a pessoas mais propensas a demonstrar altruísmo, gentileza e comportamentos pró-sociais (Ashton, 2013). Essas pessoas, frequentemente, prezam por bons relacionamentos interpessoais no seu ambiente de trabalho e tendem a obter maior suporte social por parte dos seus pares, o que, muitas vezes, funciona como fator protetivo contra o burnout (You, Huang, Wang, \& Bao, 2015). Por fim, a Conscienciosidade é marcada por características que incluem organização, perfeccionismo e disciplina, autocontrole e responsabilidade no cumprimento de objetivos e tarefas (Ashton, 2013). Este traço está muito relacionado à capacidade e vontade de trabalhar com empenho, zelo e ao bom desempenho profissional (Heineck \& Anger, 2010). Ainda não há consentimento sobre o quanto este traço é capaz de predizer o burnout, mas resultados obtidos em metanálises de diferentes países apontam para uma possível influência de aspectos culturais (Swider \& Zimmerman, 2010; You et al., 2015).

\section{Variáveis sociodemográficas e o Burnout}

Variáveis sociodemográficas, como idade, sexo e escolaridade, por exemplo, vêm sendo associadas ao burnout (Marinaccio et al., 2013). No entanto, os resultados encontrados mostram-se inconsistentes, seja pelas diferenças metodológicas adotadas ou por diferenças regionais e culturais (Campos et al., 2015).

Pesquisas apontam que as mulheres tendem a experienciar maiores níveis de sintomas de ansiedade, estresse e depressão quando comparadas aos homens (Arnten, Jansson, \& Archer, 2008). Segundo Bostjancic, Kocjan e Stare (2015), estudos prévios sobre o burnout apontam para a existência de diferenças entre homens e mulheres, mas os resultados não são definitivos. Comumente, mulheres apresentam maiores níveis de Exaustão enquanto homens, de Despersonalização (Shirom, 2003; Maslach \& Leiter, 2016). Todavia, algumas pesquisas mostram o contrário, sugerindo, inclusive, que o tipo de profissão e políticas de fomento à igualdade de gêneros na organização influenciam a não diferenciação dos níveis de burnout entre os sexos (Aguayo, Vargas, Cañadas, \& De La Fuente, 2017; Purvanova \& Muros, 2010; Taka et al., 2016).

Quanto a variável idade, acredita-se que pessoas mais velhas possuem mais tempo de experiência profissional e, com isso, tendem a apresentar maior repertório de estratégias para enfrentar o estresse e adversidades do trabalho (Bostjancic et al., 2015). No entanto, em revisão sistemática (Campos et al., 2015) sobre o burnout em profissionais de enfermagem, foram encontrados estudos que apontavam para relação positiva entre idade e os fatores do burnout, ou seja, profissionais mais velhos estariam mais vulneráveis.

Trabalhadores com maior nível educacional são retratados como mais suscetíveis ao burnout pela literatura (Holmes, Alves, Holmes, Viana, \& Santos, 2014), possivelmente por estas pessoas geralmente assumirem cargos de maior importância e responsabilidade ou pelo descontentamento com o fato de o trabalho não atender às suas expectativas (Maslach et al., 2001). Estes resultados chamam atenção, já que níveis mais elevados de estresse no trabalho estão mais associados a profissionais menos qualificados (Lunau, Siegrist, Dragano, \& Wahrendorf, 2015; Marinaccio et al., 2013).

Diante do exposto, a presente pesquisa teve por objetivo investigar a relação entre variáveis individuais, aqui representadas pelos traços de personalidade e variáveis sociodemográficas, e profissionais sobre o desfecho do burnout. Para tal, foi selecionada uma amostra composta por trabalhadores brasileiros do setor terciário da economia. 


\section{Método}

A presente pesquisa faz parte de um projeto mais amplo que tem por finalidade desenvolver e buscar evidências de validade de uma nova medida para avaliação do burnout, intitulada de Inventário de Burnout no Trabalho (IBT, Damásio \& Borsa, 2017) e construída para o contexto brasileiro. O método utilizado para este estudo foi o correlacional para se compreender melhor a relação entre variáveis individuais no desfecho do burnout, uma vez que a literatura carece de dados conclusivos sobre isso.

\section{Participantes}

A amostra, do tipo não probabilística, contou com 343 trabalhadores de todas as regiões do país. Com relação às características sociodemográficas, $n=$ $259(75,50 \%)$ dos participantes declararam ser do sexo feminino e as idades variaram dos 19 aos 63 anos $(M$ $=35,23 ; D P=9,07)$. Os participantes avaliados eram profissionais do terceiro setor da economia (e.g. profissionais da saúde, educação, serviços administrativos, segurança). Nesta pesquisa, foram avaliados profissionais de todas as regiões do território nacional, sendo $n=167(48,68 \%)$ do Nordeste, $n=72(21,00 \%)$ do Sudeste, $n=60(17,49 \%)$ do Sul, $n=30(8,75 \%)$ do Centro-Oeste e $n=14$ (4,08\%) do Norte. Quanto ao nível de escolaridade, predominaram os profissionais com Pós-Graduação $(57,10 \%, n=196)$, seguidos por Ensino Superior Completo $(28,60 \%, n=98)$, Ensino Superior Incompleto $(9,90 \%, n=34)$, Ensino Médio Completo $(3,20 \%, n=11)$ Ensino Médio Incompleto $(0,9 \%, n=3)$ Ensino Fundamental Completo $(0,3 \%, n$ $=1)$. Com relação à renda mensal, a maioria dos profissionais possuía renda superior a 10 salários mínimos $(35,32 \% ; n=121)$, seguidos por aqueles com renda entre 5 e 10 salários $(34,40 \% ; n=118)$ e aqueles com renda entre 1 e 5 salários $(30,28 \% ; n=104)$.

\section{Instrumentos}

Questionário sociodemográfico: foi utilizado um questionário sociodemográfico de autorrelato e estruturado para coletar os seguintes dados demográficos: sexo, idade, escolaridade, profissão, renda, horas de trabalho, horas extras trabalhadas, carga de trabalho e localidade de residência.

Inventário de Burnout no Trabalho (IBT, Damásio \& Borsa, 2017): este instrumento foi elaborado a partir do modelo de três dimensões do burnout proposto por Maslach e Jackson (1981): Exaustão Emocional,
Despersonalização e Baixa Realização no Trabalho. O processo de construção do IBT foi conduzido segundo proposta de Damásio e Borsa (2017) que abarca diferentes etapas incluindo a conceituação do construto (definição constitutiva e operacional), a elaboração dos itens e a análise qualitativa (avaliação por juízes e público alvo) e quantitativa dos itens (análise fatorial exploratória e confirmatória e consistência interna). $\mathrm{O}$ IBT é um instrumento composto por 25 itens, sendo 9 de Exaustão Emocional (e.g. "Estou me sentindo emocionalmente esgotado(a) no meu trabalho"), 8 de Despersonalização (e.g. "Não tenho conseguido ser atencioso com as pessoas as quais preciso lidar no meu trabalho") e 8 de Baixa Realização no Trabalho (e.g. "Tenho sentido que o meu trabalho não vale a pena"). É composto por uma escala Likert de 5 pontos $(1=$ Discordo fortemente a $5=$ Concordo fortemente $)$. Nos estudos iniciais de validade (Damásio \& Borsa, 2017), o instrumento apresentou carga fatorial variando de 0,64 (Baixa Realização no Trabalho) a 0,90 (Exaustão Emocional) e bons índices de ajuste para modelo de segunda ordem $\left(\chi^{2}=1032,5, p<0,001 ; \chi^{2} / g 1=3,79\right.$; RMSEA $=0,08$; CFI $=0,97$; TLI $=0,96$ ). Quanto aos índices de confiabilidade, todas as dimensões apresentaram valores para o alpha de Chronbach adequados, variando de $\alpha=0,90$ (Baixa Realização no Trabalho) a $\alpha=0,94$ (Exaustão Emocional).

Marcadores Redu₹idos de Personalidade (Hauck-Filho, Machado, Teixeira, \& Bandeira, 2012): esse instrumento, baseado no modelo dos Cinco Grande Fatores de Personalidade é composto por 25 adjetivos divididos que complementam o enunciado "Eu sou uma pessoa..." com uma escala Likert de 5 pontos $(1=$ Discordo totalmente e 5 = Concordo totalmente). Os autores encontraram índices de consistência interna variando de $\alpha=$ 0,78 (Abertura à experiência) a $\alpha=0,83$ (Extroversão).

\section{Procedimento de coleta dos dados}

Os profissionais foram selecionados a partir dos critérios do Relatório da Classificação Nacional de Atividades Econômicas (IBGE, 2007). Desta forma, a lista de áreas profissionais criadas para esta pesquisa compreende 16 subsetores, a saber: profissionais do comércio, turismo e lazer, educação, restaurantes, profissionais da saúde, bancos e consultoria financeira, transportes e serviços de entrega, corretagem de imóveis, assistência social, assistência técnica em geral, atendimento (pessoal, telemarketing, call-centers etc.), serviços administrativos ou jurídicos, marketing e publicidade, segurança pública ou privada, serviços 
relacionados ao setor de informação e serviços de arquitetura, engenharia, testes e análises técnicas. As profissões representadas por número igual ou menor a 20 indivíduos foram agrupadas em uma nova categoria chamada "Outras Profissões". Dentre as áreas inseridas nesta categoria estão, por exemplo: atendimento (e.g. pessoal, telemarketing) e corretagem de imóveis.

Para colaborarem com o estudo, os participantes foram convidados por e-mail ou por meio de redes sociais diversas (e.g. Facebook) e solicitados a responderem e indicarem o questionário para outras pessoas (Snowball sampling, Patton, 2002). Foram informados sobre a não-obrigatoriedade da participação, sendo que quem optou por participar, respondeu ao questionário por meio de uma plataforma online. As primeiras páginas do questionário continham uma breve apresentação do projeto e o Termo de Consentimento Livre e Esclarecido (TCLE). Por se tratar de uma pesquisa envolvendo seres humanos, os dados foram coletados após aprovação do Conselho de Ética em Pesquisa do Centro de Filosofia e Ciências Humanas $(\mathrm{CFCH})$ da Universidade Federal do Rio de Janeiro (UFRJ), sob número CAEE 62340016.0.0000.5582.

\section{Procedimento de análise dos dados}

Inicialmente, foram realizadas análises descritivas (média, frequência e porcentagem) das variáveis sociodemográficas da amostra para melhor conhecimento da mesma. Para analisar as diferenças das médias entre os fatores do burnout e as variáveis sexo e escolaridade (Profissionais até Ensino Superior Incompleto e Profissionais com Ensino Superior Completo e Pós-Graduação) foi conduzida uma MANOVA.

A partir dos escores totais das variáveis avaliadas, verificou-se que o pressuposto de normalidade da amostra não foi atendido (Kolmogorov-Smirnov, $p<$ 0,001; Shapiro-Wilk, $p<0,001)$. A solução para este problema foi a utilização do procedimento de bootstrapping (Efron, 1979), que visa aproximar a distribuição da amostra de uma distribuição normal. Para tal, foi adotado método de reamostragem simples, número de amostras $N=1000$, nível do intervalo de confiança de 95\% e tipo de intervalo de confiança corrigido e acelerado por viés (BCa). Após, foram realizadas análises de correlação bivariada de Spearman entre os traços de personalidade e os fatores de burnout, entre as variáveis sociodemográficas e os fatores do burnout e entre as variáveis laborais e os fatores do burnout. Foi utilizado o software Statistical Package for the Social Sciences (SPSS), versão 23, para a realização de tais análises.
Para analisar melhor os resultados das relações entre as variáveis, uma modelagem por equações estruturais foi implementada. Nesta pesquisa, as variáveis profissionais e sociodemográficos foram inseridas para controlar as variáveis individuais (traços de personalidade). A modelagem por equações estruturais foi realizada por meio da versão 7.11 do software Mplus, utilizando o método de estimação Weighted Least Squares Mean and Variance Adjusted (WLSMV), o qual é útil para dados ordinais não distribuídos normalmente. Para avaliar o modelo global, foram considerados os índices de ajuste Root Mean Square Error of Approximation (RMSEA), Comparative Fit Index (CFI) e Tucker-Lewis Index (TLI). Segundo a literatura, o valor do RMSEA deve ser inferior a 0,08 (com intervalo de confiança de $90 \%$ inferior a 0,10 ), enquanto os valores de CFI e TLI devem ser maiores que 0,95 (Brown, 2006).

\section{Resultados}

Os dados apresentados na Tabela 1 e 2 são referentes aos coeficientes de correlação bivariada de Spearman entre as variáveis analisadas. Das variáveis sociodemográficas analisadas, apenas a escolaridade apresentou relação estatisticamente significativa com as três dimensões do burnout, sendo esta relação fraca e negativa (Tabela 1). Com relação aos fatores contextuais, observou-se que renda, quantidade de horas trabalhadas semanalmente e carga de trabalho apresentaram relações estatisticamente significativa com os três fatores do burnout. Renda apresentou relação fraca e negativa com os três fatores e as horas de trabalho. Enquanto carga de trabalho apresentou maior magnitude e relação positiva com o burnout (Tabela 1). Quanto aos traços de personalidade, os coeficientes de correlação apontaram relação negativa e estatisticamente significativa entre os três fatores do burnout e os traços de Extroversão, Socialização e Conscienciosidade, e positiva e estatisticamente significativa com Neuroticismo. Vale ressaltar que Neuroticismo apresentou os coeficientes de maior magnitude. O único traço de personalidade que não apresentou relação significativa com o burnout foi a faceta Abertura à experiência (Tabela 2).

Uma MANOVA foi realizada para avaliar a hipótese de que haveria diferenças das médias dos níveis de burnout entre os sexos e entre os níveis de escolaridade desta amostra. O teste de igualdade de covariância de Box demonstrou que os dados apresentavam homogeneidade de variância (Box $\mathrm{M}=24,27, p=$ 
Tabela 1.

Correlação de Spearman entre as variáveis sociodemográficas, contextuais e os fatores do burnout

\begin{tabular}{lccccccccc}
\hline Variável & 1 & 2 & 3 & 4 & 5 & 6 & 7 & 8 & 9 \\
\hline 1. EE & 1 & & & & & & & & \\
2. DP & $0,74^{* *}$ & 1 & & & & & & & \\
3. BRT & $0,82^{* *}$ & $0,70^{*}$ & 1 & & & & & & \\
4. Idade & 0,03 & 0,06 & 0,16 & 1 & & & & & \\
5. Escolaridade & $-0,16^{* *}$ & $-0,19^{* *}$ & $-0,16^{* *}$ & $0,18^{* *}$ & 1 & & & & \\
6. Renda & $-0,19^{* *}$ & $-0,26^{* *}$ & $-0,22^{* *}$ & $0,19^{* *}$ & $0,35^{* *}$ & 1 & & & \\
7. Horas de trabalho & $0,18^{* *}$ & $0,20^{* *}$ & $0,12^{*}$ & 0,05 & 0,02 & $0,16^{* *}$ & 1 & & \\
8. Horas extras & 0,08 & 0,05 & 0,03 & $-0,07$ & 0,07 & 0,08 & $0,18^{* *}$ & 1 \\
9. Carga & $0,38^{* *}$ & $0,19^{* *}$ & $0,13^{*}$ & $0,16^{* *}$ & 0,06 & 0,06 & 0,05 & $0,17^{* *}$ & 1 \\
\hline
\end{tabular}

Nota. EE = Exaustão Emocional; DP = Despersonalização; BRT = Baixa Realização no Trabalho; Carga = Carga de trabalho; Correlação com ausência de asterisco indica efeito não significativo; ${ }^{*} p<0,05 ;{ }^{* *} p<0,01$.

Tabela 2.

Correlaşão de Spearman entre os traços de personalidade e os fatores do burnout

\begin{tabular}{|c|c|c|c|c|c|c|c|c|}
\hline Variável & 1 & 2 & 3 & 4 & 5 & 6 & 7 & 8 \\
\hline 1. EE & 1 & & & & & & & \\
\hline 2. DP & $0,74^{* *}$ & 1 & & & & & & \\
\hline 3. BRT & $0,82^{* *}$ & $0,70^{*}$ & 1 & & & & & \\
\hline 4. Neuroticismo & $0,57^{* *}$ & $0,48^{* *}$ & $0,54^{* *}$ & 1 & & & & \\
\hline 5. Extroverção & $-0,23^{* *}$ & $-0,21^{* *}$ & $-0,19^{* *}$ & $-0,41^{* *}$ & 1 & & & \\
\hline 6. Socialização & $-0,17^{* *}$ & $-0,36^{* *}$ & $-0,19^{* *}$ & $-0,24^{* *}$ & $0,21^{* *}$ & 1 & & \\
\hline 7. Consci. & $-0,12^{*}$ & $-0,28^{* *}$ & $-0,18^{* *}$ & $-0,17^{* *}$ & $0,11^{*}$ & $0,62^{* *}$ & 1 & \\
\hline 8. Abertura & $-0,01$ & $-0,04$ & $-0,04$ & $-0,18^{* *}$ & $0,26^{* *}$ & $0,23^{* *}$ & $0,18^{* *}$ & 1 \\
\hline
\end{tabular}

Nota EE = Exaustão Emocional; DP = Despersonalização; BRT = Baixa Realização no Trabalho; Consci. = Conscienciosidade; Correlação com ausência de asterisco indica efeito não significativo; ${ }^{*} p<0,05 ;{ }^{* *} p<0,01$.

0,185). No entanto, não foram encontradas diferenças estatisticamente significativas entre os sexos $[\mathrm{V}=0,014$, $F(3,337)=1,58, p=0,19]$, nem entre os níveis de escolaridade $[\mathrm{V}=0,004, F(3,337)=0,41, p=0,74]$ e os fatores de burnout (Tabela 3).

Em seguida, foi realizada uma modelagem por equações estruturais para compreender a relação entre as variáveis individuais de personalidade, os fatores sociodemográficos e contextuais sobre o burnout chegando-se a um modelo final satisfatório $\left(\chi^{2}=2586,98\right.$; $g l=1476 ; \chi^{2} / g l=1,75 ;$ CFI $=0,96 ;$ TLI $=0,96$; RMSEA (I.C. $90 \%)=0,047$ [0,045, 0,051]), sugerindo que o modelo é adequado (Figura 2). Neste modelo, foi demonstrado que os traços de personalidade foram as variáveis de maior influência sobre o desfecho do burnout. Dos traços de personalidade, Neuroticismo apresentou relação estatisticamente significativa e positiva com os três fatores da síndrome $\left(\beta_{\mathrm{FE}}=0,60, p\right.$ $\left.<0,001 ; \beta_{\mathrm{DP}}=0,48, p<0,001 ; \beta_{\mathrm{BRT}}=0,62, p<0,001\right)$, além de ter sido a variável que apresentou maior magnitude nas relações. $\mathrm{O}$ traço de Abertura à experiência, também apresentou relação estatisticamente significativa e positiva com as três dimensões do burnout $\left(\beta_{\mathrm{EE}}\right.$ $=0,14, p<0,01 ; \beta_{\mathrm{DP}}=0,11, p<0,05 ; \beta_{\mathrm{BRT}}=0,13, p$ $<0,05)$. Já o traço de Socialização apresentou relação estatisticamente significativa e negativa apenas com a dimensão da Despersonalização $\left(\beta_{\mathrm{DP}}=-0,32, p<\right.$ $0,001)$. Com relação às variáveis de controle, inseridas no modelo para controlar os efeitos das variáveis de personalidade, apenas as variáveis profissionais apresentaram relação significativa com os fatores do burnout. A renda desempenhou relação negativa com os três fatores do burnout $\left(\beta_{\mathrm{EE}}=-0,23, p<0,001 ; \beta_{\mathrm{DP}}=-0,33\right.$, $\left.p<0,001 ; \beta_{\text {BRT }}=-0,25, p<0,001\right)$, enquanto horas de 
trabalho $\left(\beta_{\mathrm{EE}}=0,20, p<0,001 ; \beta_{\mathrm{DP}}=0,27, p<0,001\right.$; $\left.\beta_{\mathrm{BRT}}=0,17, p<0,001\right)$ e carga de trabalho $\left(\beta_{\mathrm{EE}}=0,32\right.$, $\left.p<0,001 ; \beta_{\mathrm{DP}}=0,17, p<0,001\right)$ apresentaram relação positiva. As variáveis sexo, idade e escolaridade não apresentaram relação significativa com o burnout neste modelo (Figura 1).

\section{Discussão}

Com base nos dados analisados, observou-se que não houve diferença estatisticamente significativa entre a variável sexo e os fatores do burnout. Vale salientar que a literatura apresenta informações contraditórias

Tabela 3.

Comparação entre as médias dos fatores do burnout e a escolaridade

\begin{tabular}{lcccc}
\hline Fatores & Escolaridade & $M$ & $D P$ & I.C. 95\% \\
\hline \multirow{2}{*}{ Exaustão Emocional } & Grupo I & 3,51 & 0,17 & {$[3,19-3,85]$} \\
& Grupo II & 3,33 & 0,07 & {$[3,18-3,47]$} \\
\multirow{2}{*}{ Despersonalização } & Grupo I & 3,14 & 0,16 & {$[2,82-3,46]$} \\
& Grupo II & 2,96 & 0,07 & {$[2,82-3,10]$} \\
\multirow{2}{*}{ Baixa Realização no Trabalho } & Grupo I & 3,41 & 0,17 & {$[3,08-3,74]$} \\
& Grupo II & 3,25 & 0,07 & {$[3,11-3,39]$} \\
\hline
\end{tabular}

Nota. Grupo I = Profissionais com até Ensino Superior Incompleto; Grupo II = Profissionais com Ensino Superior Completo e Pós-Graduação; $M$ = Média; $D P=$ Desvio-padrão; I.C. = Intervalo de Confiança.

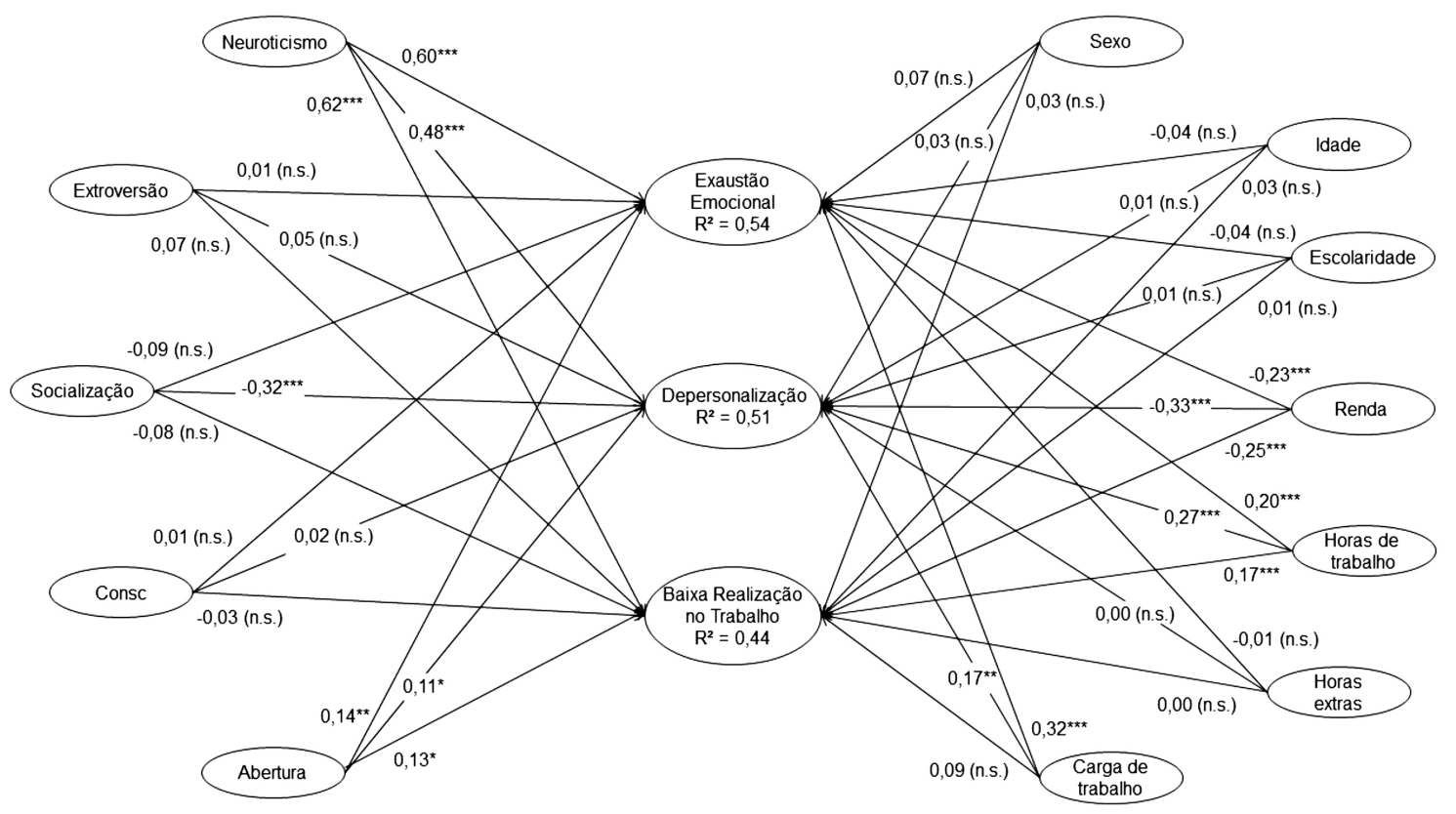

${ }^{*} p<0,05 ;{ }^{* * *} p<0,01 ;{ }^{* * *} p<0,001$.

Figura 1. Relação entre as variáveis profissionais (sexo, idade, escolaridade, renda, horas de trabalho, horas extras e carga de trabalho) e os traços de personalidade (Neuroticismo, Extroversão, Socialização, Conscienciosidade e Abertura à experiência) sobre o desfecho dos três fatores do burnout (Exaustão Emocional, Despersonalização e Baixa Realização no Trabalho) 
quanto a esse aspecto (Heinemann \& Heinemann, 2017). Em geral, observam-se maiores níveis de Exaustão Emocional em mulheres e de Despersonalização em homens (Bostjancic et al., 2015). Porém, segundo metanálise realizada por Purvanova e Muros (2010), os tamanhos de efeito encontrados nas comparações entre cada um dos dois fatores centrais do burnout (Exaustão Emocional e Despersonalização) e a variável sexo foram pequenos. Muitas vezes, esta diferença de resultados, quando presente, pode ser explicada por outros motivos, como o efeito do viés cultural (Maslach et al., 2001; Purvanova \& Muros, 2010), condições de trabalho (Taka et al., 2016) e presença ou ausência de suporte social na organização (Rivera-Torres, Araque-Padilla, \& Montero-Simó, 2013).

Não foram observadas diferenças estatisticamente significativas das médias dos fatores do burnout nas diferentes faixas etárias. Embora alguns autores reportem uma maior incidência do burnout em profissionais mais jovens (Bostjancic et al., 2015), seja pela imaturidade, falta de experiência profissional ou pouco repertório de estratégias para lidar com os estressores no trabalho (Gomez-Urquiza, Vargas, De La Fuente, Fernandez-Castillo, \& Canadas-De La Fuente, 2017), estudos apontam para a uma baixa relevância da idade como preditor do burnout (Aguayo et al., 2017). Quando o ambiente organizacional é permeado de estressores, os recursos utilizados pelos funcionários, experientes ou não, podem não ser suficientes para que eles lidem com os afetos negativos gerados pelo trabalho (Aguayo et al., 2017). É sabido que em cenários de crise econômica, há maior tendência ao adoecimento psíquico dos funcionários, seja pelo medo de demissão, aumento de sobrecarga de trabalho ou perda de benefícios (Maslach \& Leiter, 2016). O fato de o cenário econômico brasileiro estar passando por transformações nos últimos anos talvez impeça que essa diferença seja observada, já que tais características impactam indivíduos de ambos os sexos e de todas as faixas etárias.

A variável referente ao nível de escolaridade também não apresentou relações estatisticamente significativas com os fatores do burnout. Alguns autores sugerem que profissionais mais qualificados tendem a sofrer maiores níveis de burnout, já que, frequentemente, assumem posições de maior responsabilidade e exigência (Marinaccio et al., 2013). Todavia, não há um consenso na literatura (Lunau et al., 2015), uma vez que outros estudos apontam que profissionais com baixa escolaridade estão mais sujeitos a piores condições de trabalho e menor bem-estar no trabalho, o que também poderia justificar níveis elevados de burnout (Kahneman \& Deaton, 2010; Shanafelt et al., 2012).

Com relação às variáveis de controle, a modelagem por equações estruturais demonstrou que as variáveis profissionais apresentaram maior relação preditiva com o burnout quando comparadas às variáveis sociodemográficas. Renda, quantidade de horas trabalhadas e carga de trabalho mostraram-se variáveis mais relevantes no desfecho da síndrome que as variáveis sociodemográficas, corroborando com o que a literatura (Aguayo et al., 2017). Em contextos laborais em que a organização não prioriza o equilíbrio entre as demandas e os recursos por ela oferecidos, é praticamente inevitável que o ambiente de trabalho não seja adoecedor. Variáveis como a sobrecarga de trabalho, jornadas longas de trabalho e baixos salários são apenas alguns exemplos, avaliados na presente pesquisa, que podem desestimular o trabalho, favorecer conflitos e facilitar o processo de esgotamento dos funcionários (Hu \& Schaufeli, 2011; Mchugh \& Ma, 2014).

No que diz respeito aos traços de personalidade, os dados obtidos apontam para o Neuroticismo como principal fator de predição das três dimensões do burnout. O Neuroticismo está associado a indivíduos com menor energia e vitalidade, maior tendência à procrastinação e a interpretar os eventos da vida de forma negativa e pessimista (Ashton, 2013). A alta relação entre Neuroticismo e Exaustão Emocional sugere que indivíduos com altos escores nesse traço são mais suscetíveis a sentirem-se esgotados pelas obrigações do trabalho (Swider \& Zimmerman, 2010; You et al., 2015). Ademais, sua relação com Despersonalização tem a ver com o aspecto da instabilidade emocional, irritabilidade e impulsividade presentes em altos níveis desse traço, podendo levar a comportamentos de cinismo, agressividade e distanciamento emocional para com os pares no ambiente de trabalho e clientes (Queirós, Kaiseler, \& Silva, 2013). Outro fato importante é a inclinação à fragilidade emocional e baixa autoestima associadas a esse traço (Ashton, 2013) e que podem influenciar na interpretação inadequada do indivíduo sobre seu desempenho, gerando redução de sentimento de realização profissional (Bakker, Van Der Zee, Lewig, \& Dollard, 2006).

É sabido que a dimensão da Exaustão Emocional exerce papel central no processo de desenvolvimento do burnout, sendo preditor das dimensões de Despersonalização e Baixa Realização no Trabalho (Maslach \& Leiter, 2016). O fato de o Neuroticismo ter apresentado maior magnitude na relação com o fator da Exaustão é 
um importante dado para se pensar como uma variável de crucial importância para o desfecho da síndrome. Mesmo que outros aspectos possam estar associados, como condições inadequadas de trabalho, altos níveis de Neuroticismo estão ligados a maior propensão a subestimar seu próprio desempenho profissional e a apresentar fortes reações emocionais em situações estressantes (Bakker et al., 2006), indivíduos com altos níveis de Neuroticismo podem ter seu desgaste físico, cognitivo e emocional potencializado ou, até mesmo, acelerar o processo de esgotamento, distanciamento e redução do senso de auto eficácia, típicos do burnout.

Indivíduos com altos escores de Socialização, normalmente, tendem a ser mais cuidadosos e compreensivos, dando atenção ao bem-estar e às necessidades dos outros, além de perceberem melhor o suporte social, contribuindo para o manejo das tensões no trabalho (Huang et al., 2014). Portanto, é menos provável que indivíduos com tal perfil lidem com as outras pessoas de forma fria e emocionalmente distante (You et al., 2015). De tal forma, pode-se considerar uma consequência que esta característica de personalidade esteja relacionada negativamente ao fator de Despersonalização (Swider \& Zimmerman, 2010).

Por fim, o fator de Abertura à experiência apresentou relação estatisticamente significativa, fraca e positiva com a Exaustão Emocional e Despersonalização, contrariando os achados da literatura (Swider \& Zimmerman, 2010). Pessoas com altos índices neste traço tendem a serem mais abertas a novas ideias, discussões e a busca por soluções mais criativas dos problemas cotidianos (Ashton, 2013). Os dados encontrados nesta pesquisa talvez possam ser indicativos de que a relação entre as organizações e os trabalhadores avaliados nesta pesquisa não contemplam soluções criativas ou, por vezes, não contribuam para a autonomia dos mesmos, fato este que está intimamente ligado ao agravamento de estressores no trabalho (Byron, Khazanchi, \& Nazarian, 2010).

É importante frisar que o traço de Abertura à experiência costuma apresentar relação fraca com os fatores do burnout, sugerindo que as características contextuais, como aspectos socioculturais, possam exercer influência nessa relação (Swider \& Zimmerman, 2010). Outro viés a ser destacado é que indivíduos com altos índices de Abertura à experiência tendem a estar mais abertos a experienciarem novas possibilidades e a buscarem opções de resolução de problemas que talvez pessoas com outros perfis de personalidade tenham mais receio em executar como, por exemplo, buscarem outros locais de trabalho ou até mesmo mudarem de profissão.

Com base no exposto acima, é possível afirmar que os traços de personalidade podem desempenhar um papel importante no desenvolvimento dos sintomas do burnout, atuando como preditores mais significativos que a idade, sexo e escolaridade. Especificamente, o Neuroticismo foi a característica de personalidade mais importante na predição dos fatores da síndrome e, portanto, pode ser considerado um fator de risco a ser levado em conta. Esse resultado está em consonância com os estudos que buscam compreender a relação entre os fatores de personalidade e os sintomas de burnout (Swider \& Zimmerman, 2010; You et al., 2015).

A presente pesquisa teve por objetivo analisar as relações entre os traços de personalidade, variáveis sociodemográficos e os fatores do burnout numa amostra de trabalhadores brasileiros do setor terciário. Além disso, buscou-se analisar o quanto os traços de personalidade responderiam como preditores de cada uma das três dimensões do burnout. A partir dos resultados obtidos, foi possível evidenciar a importância das características individuais sobre o burnout, sendo os traços de personalidade as variáveis preditivas que mais se destacaram no modelo testado.

Ademais, corroborou-se dados prévios da literatura que apontam para o Neuroticismo como um traço importante a ser analisado para se compreender o desgaste emocional no trabalho (Dalanhol et al., 2017). Vale ressaltar que os dados aqui apresentados não têm por objetivo atrelar ao indivíduo a causa para o desgaste emocional oriundo do trabalho, já que é conhecido o impacto das características laborais no adoecimento psíquico do trabalhador (Maslach \& Leiter, 2016). No entanto, é importante que se compreenda melhor o papel dos traços de personalidade no adoecimento psicológico, aqui representado pelo burnout, para se pensar em estratégias de intervenção e educação que contribuam para a promoção de qualidade de vida nas empresas. Outro aspecto a ser frisado é o da necessidade de mais pesquisas que investiguem a importância de se considerar os traços de personalidade na seleção profissional com base no perfil exigido para a vaga ou cargo (Nikolaou \& Foti, 2017). Nunca pensando com um viés segregacionista, mas em como contribuir para o melhor aproveitamento das potencialidades e talentos de cada indivíduo.

Embora os resultados tenham sido importantes para a realidade brasileira, esta pesquisa também apresentou limitações no que tange ao viés elitista da 
amostra, uma vez que a maior parte dos respondentes apresentavam pós-graduação e renda familiar superior a 10 salários mínimos. Para uma melhor compreensão das relações aqui apresentadas, sugere-se novas investigações focadas nas características específicas de cada grupo profissional e que considerem outros perfis sociodemográficos, incluindo participantes de outros estados e regiões do Brasil. Outro aspecto que deve ser salientado é a necessidade de realização de novas pesquisas com grupos clínicos e que possam elucidar melhor a relação do burnout com características individuais e laborais.

\section{Referências}

Aguayo, R., Vargas, C., Cañadas, G., \& De la Fuente, E. (2017). Are socio-demographic factors associated to burnout syndrome in police officers. Anales de Psicologia, 33(2), 383-392. doi: 10.6018/ analesps.33.2.260391.

Arnten, A. A., Jansson, B., \& Archer, T. (2008). Influence of affective personality type and gender upon coping behavior, mood, and stress. Individual Differences Research, 6(3), 139-168.

Ashton, M. C. (2013). Individual differences and personality. Ontário: Academic Press.

Bakker, A. B., Demerouti, E., \& Sanz-Vergel, A. I. (2014). Burnout and work engagement: The JD-R approach. Annual Review of Organizational Psychology and Organizational Behavior, 1, 389-411. doi: 10.1146/annurev-orgpsych-031413-091235.

Bakker, A. B., Van Der Zee, K. I., Lewig, K., \& Dollard, M. (2002). The relationship between the big-five personality factors and burnout: A study among volunteer counselors. The Journal of Social Psychology, 146(1), 31-50. doi: 10.3200/socp.146.1.31-50.

Bostjancic, E., Kocjan, G. Z., \& Stare, J. (2015). Role of socio-demographic characteristics and working conditions in experiencing burnout. Suvremena psibologija, 18(1), 43-60.

Brown, T. A. (2006). Confirmatory factor analysis for applied research. New York: Guilford.

Byron, K., Khazanchi, S., \& Nazarian, D. (2010). The relationship between stressors and creativity: A meta-analysis examining competing theoretical models. Journal of Applied Psychology, 95(1), 201-212. doi: $10.1037 /$ a0017868
Campos, I. C. M., Angélico, A. P., Oliveira, M. S., \& Oliveira, D. C. R. (2015). Fatores sociodemográficos e ocupacionais associados à síndrome de burnout em profissionais de enfermagem. Psicologia Reflexão e Crítica, 28(4), 764-771. doi: 10.1590/1678-7153.201528414

Carlotto, M. S. (2011). Síndrome de burnout em professores: Prevalência e fatores associados. Psicologia: Teoria e Pesquisa, 27(4), 139-147. doi: 10.1590/ s0102-37722011000400003

Dalanhol, N. S., Freitas, C. P. P., Machado, W. L., Hutz, C. S., \& Vazquez, A. C. S. (2017). Engajamento no trabalho, saúde mental e personalidade em oficiais de justiça. Psico, 48(2), 109-119. doi: 10.15448/1980-8623.2017.2.25885

Damásio, B. F., \& Borsa, J. C. (2019). Manual do Inventário de Burnout no Trabalho. Manuscrito não publicado.

Demerouti, E., Nachreiner, F., Bakker, A. B., \& Schaufeli, W. B. (2001). The job demand-resources model of burnout. Journal of Applied Psychology, 86(3), 499-512.

Efron, B. (1979). Bootstrap methods - Another look at the jackknife. The annals of statistics, 7(1), 1-26. doi: 10.1007/978-1-4612-4380-9_41

Freudenberger, H. J. (1974). Staff burn-out. Journal of Social Issues, 30(1), 159-165. doi: 10.1111/j.15404560.1974.tb00706.x

Gomez-Urquiza, J. L., Vargas, C., De la Fuente, E., Fernandez-Castillo, R., \& Canadas-De la Fuente, G. A. (2017). Age as a risk factor for burnout syndrome in nursing professionals: A meta-analytic study. Research in Nursing \& Health, 40, 99-110. doi: $10.1002 /$ nur. 21774

Hauck-Filho, N., Machado, W., Teixeira, M. A. P., \& Bandeira, D. R. (2012). Evidências de validade de marcadores reduzidos para a avaliação da personalidade no modelo dos cinco grandes fatores. Psicologia: Teoria e Pesquisa, 28(4), 417-423. doi: 10.1590/S0102-37722012000400007

Heineck, G., \& Anger, S. (2010). The returns to cognitive abilities and personality traits in Germany. Labour Economics, 17, 535-546. doi: 10.1016/j. labeco.2009.06.001

Heineman, L. V., \& Heineman, T. (2017). Burnout research: Emergence and scientific investigation of

Psico-USF, Bragança Paulista, v. 25, n. 3, p. 439-450, jul./set. 2020 
a contested diagnosis. SAGE Open, 7(1), 1-12. doi: $10.1177 / 2158244017697154$

Holmes, E., Alves, J., Holmes, D., Viana, Y., \& Santos, S. (2014). Síndrome de burnout em enfermeiros da Estratégia Saúde da Família. Revista de Enfermagem UFPE, 8(7), 1841-1847. doi:10.5205/ reuol.5963-51246-1-RV.0807201402

Huang, J. L., Ryan, A. M., Zabel, K. L., \& Palmer, A. (2014). Personality and adaptive performance at work: A meta-analytic investigation. Journal of Applied Psychology, 99(1), 162-179. doi: 10.1037/ a0034285

Instituto Brasileiro de Geografia e Estatística (2007). Classificação Nacional de Atividades Econômicas. In Comissão Nacional de Classificaşão. Recuperado de https://cnae.ibge.gov.br/?view=estrutura.

Judge, T. A., Higgins, C. A., Thoresen, C. J., \& Barrick, M. R. (1999). The big-five personality traits, general mental ability, and career success across the life span. Personnel Psychology, 52(3), 621-652. doi: 10.1111/j.1744-6570.1999.tb00174.x

Judge, T. A., \& Ilies, R. (2002). Relationship of personality to performance motivation: A metanalytic review. Journal of Applied Psychology, 87(4), 797-807. doi: 10.1037//0021-9010.87.4.797

Kahneman, D., \& Deaton, A. (2010). High income improves evaluation of life but not emotional well-being. PNAS, 107(38), 16489 - 16493. doi: 10.1073/pnas.1011492107

Lunau, T., Siegrist, J., Dragano, N., \& Wahrendorf, M. (2015). The association between education and work stress: Does the policy context matter? Plos One, 10(3), 1-17 doi: 1 0.1371/journal. pone. 0121573

Marinaccio, A., Ferrante, P., Corfiati, M., Di Tecco, C., Rondinone, B. M., Bonafede, M., Ronchetti, M., Persechino, B., \& Iavicoli, S. (2013). The relevance of socio-demographic and occupational variables for the assessment of workrelated stress risk. BMC Public Health, 13(1), 1-9. doi: 10.1186/1471-2458-13-1157

Maslach, C., \& Leiter, M. P. (1997). What causes burnout?. In C. Maslach, \& M. P. Leiter (Eds.), The truth about burnout: How organizations cause personal stress and what to do about it (pp. 38 -60). San Francisco, Califórnia: Wiley.
Maslach, C., \& Leiter, M. (2016). New insights into burnout and health care: Strategies for improving civility and alleviating burnout. Medical Teacher. doi: 10.1080/0142159X.2016.1248918

Maslach, C., Schaufeli, W. B., \& Leiter, P. L. (2001). Job burnout. Annual Review of Psychology, 52, 397-422. doi: 0066-4308/01/0201-0397\$14.00

Nikolaou, I., \& Foti, K (2017). Personnel selection and personality. In V. Zeigler-Hill, \& T. K. Shackelford (Eds.), The SAGE bandbook of personality and individual differences. (pp. 458 - 474). Oakland, Califórnia: SAGE Publications Ltd.

Patton, M. Q. (2002). Qualitative research \& evaluation methods. London: Sage Publications.

Purvanova, R., \& Muros, J. (2010). Gender differences in burnout: A meta-analysis. Journal of Vocational Behavior, 77(2), 168-185. doi: 10.1016/j. jvb.2010.04.006

Queirós, C., Kaiseler, M., \& Silva, L. A. (2013). Burnout as predictor of aggressivity among police officers. European Journal of Policing Studies, 1(2), 110-135.

Rivera-Torres, P., Araque-Padilla, R. A., \& MonteroSimó, M. J. (2013). Job stress across gender: The importance of emotional and intellectual demands and social support in women. International Journal of Environmental Research and Public Health, 10, 375389. doi: 10.3390/ijerph10010375

Shanafelt, T. D., Boone, S., Tan, L., Dyrbye, L. N., Sotile, W., Satele, D., West, C. P., Sloan, J., \& Oreskovich, M. R. (2012). Burnout and satisfaction with work-life balance among US physicians relative to the general US population. Archives of Internal Medicine, 172(18), 1 377-1385. doi: 10.1001/ archinternmed.2012.3199

Schaufeli, W. B., \& Taris, T.W. (2014). A critical review of the job demands-resources model: Implications for improving work and health. In G. F., Bauer, \& O., Hämmig. (Eds). Bridging occupational, organizational and public health. Amsterdam: Springer.

Shirom, A. (2003). Job-related burnout: A review. In J.C. Quick, \& L.E. Tetrick (Eds.), Handbook of occupational health psychology (pp. 245-264). Washington: APA.

Swider, B. W., \& Zimmerman, R. D. (2010). Born to burnout: A meta-analytic path model of personality, job burnout, and work outcomes. Journal of 
Vocational Behavior, 76(3), 487-506. doi: 10.1016/j. jvb.2010.01.003

Taka, F., Nomura, K., Horie, S., Takemoto, K., Takeuchi, M., Takenoshita, S., Murakami, A., Hiraike, H., Okinaga, H., \& Smith, D. R. (2016). Organizational climate with gender equity and burnout among university academics in Japan. Industrial Health, 54(6), 480-487. doi: 10.2486/indhealth.2016-0126
You, X., Huang, J., Wang, Y., \& Bao, X. (2015). Relationships between individual-level factors and burnout: A meta-analysis of chinese participants. Personality and Individual Differences, 74, 139-145. doi: 10.1016/j.paid.2014.09.048

Recebido em: 31/07/2018

Reformulado em: 14/06/2019

Aprovado em: 22/07/2019

Sobre os autores:

Vitor Hugo Loureiro Bruno Costa é Psicólogo formado pela Universidade Federal do Rio de Janeiro. Mestrado em Psicologia Clínica pela Pontifícia Universidade Católica do Rio de Janeiro. Atualmente, encontra-se atuando como Docente na Universidade Estácio de Sá.

ORCID: https://orcid.org/0000-0001-6030-3466

E-mail:vhl.psi@gmail.com

Juliane Callegaro Borsa é Professora Adjunta do Departamento de Psicologia e do Programa de Pós-Graduação em Psicologia Clínica da Pontifícia Universidade Católica do Rio de Janeiro. Mestrado em Psicologia Clínica pela Pontifícia Universidade Católica do Rio Grande do Sul. Doutorado e Pós-Doutorado pela Universidade Federal do Rio Grande do Sul. Fundadora e coordenadora do Grupo de Pesquisa em Avaliação Psicológica e do Laboratório de Avaliação Psicológica de Crianças e Adolescentes.

ORCID: https://orcid.org/0000-0001-7703-5509

E-mail: juliborsa@gmail.com

Bruno Figueiredo Damásio é Professor Adjunto do Instituto de Psicologia da Universidade Federal do Rio de Janeiro, no Departamento de Psicometria. Professor Permanente do Programa de Pós-Graduação em Psicologia IP/UFRJ. Psicólogo pela Universidade Estadual da Paraíba. Mestrado e Doutorado em Psicologia pela Universidade Federal do Rio Grande do Sul. Coordenador do Laboratório de Psicometria e Psicologia Positiva.

ORCID: https://orcid.org/0000-0002-1150-092X

E-mail: bf.damasio@gmail.com

Contato com os autores:

Rua Marquês de São Vicente 225, Edifício Cardeal Leme, 2 Andar, Sala 201, Gávea

Rio de Janeiro-RJ, Brasil

CEP: 22451-900 